\title{
30-km spontaneous-Brillouin distributed temperature sensor employing Simplex-coding and low optical input power
}

\author{
Marcelo A. Soto, Gabriele Bolognini and Fabrizio Di Pasquale \\ Scuola Superiore Sant'Anna \\ Via G. Moruzzi 1, 56124 Pisa, Italy \\ Email: m.soto@sssup.it
}

\begin{abstract}
In this work we have applied optical pulse-coding technique to spontaneous Brillouin-based distributed temperature sensor (BDTS) based on the Landau-Placzek ratio (LPR). We experimentally show that pulse-coding applied to optical time domain reflectometry (OTDR) based BDTS allows for a significant performance enhancement, avoiding the use of optical amplification. Actually, by using 127-bit Simplex coding, we achieved about 7 dB signal-to-noise ratio improvement at the receiver, allowing for temperature sensing over $30 \mathrm{~km}$-distance with temperature/spatial resolution up to $5.0 \mathrm{~K} / 40 \mathrm{~m}$ using only $10 \mathrm{~mW}$ optical peak power at the fiber input.
\end{abstract}

\section{INTRODUCTION}

In the last years, fiber optic distributed sensors have been attracting a great deal of attention due to the unique capability they offer in terms of distributed sensing mechanism with important industrial, environmental and structural monitoring applications [1]. In particular, Brillouin-based distributed optical sensors are becoming increasingly attractive for temperature and strain monitoring, due to their capacity of sensing simultaneously both such physical variables [2]. This feature represents one of the main advantages of Brillouin-based distributed temperature sensors (BDTS) compared to distributed optical sensors based on spontaneous Raman scattering. Although BDTS are characterized by higher backscattering power compared to Raman-based distributed temperature sensors (RDTS) [3], they are however characterized by a lower temperature sensitivity and they also require a more complex receiver scheme.

In general terms, Brillouin-based distributed temperature sensors can be implemented using either stimulated [4] or spontaneous Brillouin scattering [5]. While the former provides better temperature resolution, requiring a more complex detection scheme and access to both fiber ends, the latter allows for a simpler implementation, accessing to only one fiber end. However, the low temperature sensitivity of Brillouin intensity components limits the performance of spontaneous-BDTS based on the Landau-Placzek ratio (LPR), especially over long sensing ranges [5], thus also negatively impacting the resolution in combined strain and temperature sensors. Several schemes have been proposed by different authors in order to improve the performance of such sensors [5, 6]. For instance, schemes based on distributed Raman amplification [5] or Erbium-doped fiber amplifiers (EDFA) [6] can provide more accurate temperature measurements over long sensing distances.

In this work we propose the use of Simplex-coding techniques $[7,8]$ in long-range BDTS. The observed signalto-noise ratio (SNR) improvement obtained by using 127-bit Simplex coding techniques results to be greater than $7 \mathrm{~dB}$, allowing for sensing over $30 \mathrm{~km}$-distance with a temperature resolution better than $5.0 \mathrm{~K}$ and a spatial resolution of $\sim 40$ $\mathrm{m}$, when using only $10 \mathrm{~mW}$ optical peak power at the fiber input. Thus, optical pulse coding offers a high-performance cost-effective alternative to optical amplification, avoiding the use of high peak power pulses into the sensing fiber.

\section{THEORY}

The implemented BDTS is based on spontaneous Brillouin intensity measurements using coded optical time domain reflectometry (OTDR). In BDTS systems, Brillouin intensity measurements need to be normalized by the Rayleigh component to account for the fiber loss. In fact, the Landau Placzek ratio (LPR) [9], which is defined as the ratio of the Rayleigh power to the Brillouin power, is normally used for compensating the intrinsic fiber loss.

The temperature dependence of the LPR is given by the well-known formula [9]:

$$
\frac{I_{R S}}{I_{S p B S}}=\frac{T_{f}}{T}\left(\beta_{T} \rho_{0} v_{s}^{2}-1\right)
$$

where $\beta_{T}$ is the isothermal compressibility, $\rho_{0}$ is the density, $T_{f}$ is the fictive temperature, $v_{s}$ is the acoustic velocity, and $I_{R S, S p B S}$ are the intensities of Rayleigh scattering and spontaneous Brillouin scattering. In order to obtain the temperature profile along the fiber, LPR at the unknown 
temperature $T$ is compared with a LPR measured at a given reference temperature $T_{R}$, obtaining [6]:

$$
T=\frac{1}{K_{T}}\left(1-\frac{\operatorname{LPR}(T)}{\operatorname{LPR}\left(T_{R}\right)}\right)+T_{R}
$$

The temperature accuracy of an OTDR-based BDTS using the LPR is mainly determined by the signal-to-noise ratio at the receiver. The optical power at the receiver is proportional to the duration of the optical pulses launched into the sensing fiber, and therefore inversely proportional to the spatial resolution. This feature leads to a critical trade-off between spatial resolution, temperature accuracy and sensing range. In order to improve the performance of BDTS, and overcome this trade-off, several techniques, mainly based on discrete and distributed optical amplification techniques, have been proposed to increase the SNR. For instance, using an Erbium-doped fiber amplifier (EDFA) as a preamplifier [6] or distributed Raman amplification [5], the accuracy of a BDTS has been improved for long distance sensing applications.

On the other hand, coding techniques have been successfully implemented for enhancing the performance of OTDR systems [7, 8]. We have also recently demonstrated that the application of optical pulse coding to Raman-based distributed temperature sensors enhances the SNR [10], providing much better temperature resolution. One suitable code for this kind of application is based on Simplex coding $[7,8]$, which involves linear combinations of traces following the so-called S-matrix. In such a scheme, the coding process is performed by modulating the intensity of an optical source according to the different codewords which are launched down along the fiber. Decoding is carried out by simple linear operations, using the several measured coded traces.

Coding techniques provide, with the same total measurement time, a considerable SNR enhancement compared to conventional single pulse case, allowing for a more accurate spatial resolution, longer sensing ranges, better resolution on the sensing variable and/or reducing the input pulse power level or measurement time. This improvement is quantified by the coding gain, defined as the ratio of the SNR obtained with coded pulses to the SNR obtained with single pulses at a given distance, and considering same number of total acquired traces. In case of Simplex coding with a code length $L$, the coding gain $G_{C O D}$ is given by [8]:

$$
G_{C O D}=\frac{L+1}{2 \sqrt{L}}
$$

Unlike distributed Raman temperature sensors based on coded OTDR, the application of pulse coding techniques to Brillouin-based sensors underlies some aspects to take into account, which are related to the different physical characteristics of Brillouin scattering with respect to Raman scattering. Characteristics such as counter-propagating direction, narrower gain bandwidth, and the required use of narrowband sources in case of Brillouin-based sensors have in fact a strong impact on the effectiveness of coding techniques in spontaneous Brillouin-based optical sensors. This is mainly due to the reduction of the threshold for onset of stimulated Brillouin scattering, resulting the most limiting factor in that case.

\section{EXPERIMENTAL SETUP}

Fig. 1 shows the experimental setup used for the spontaneous-BDTS. A tunable external cavity laser operating at $1550.0 \mathrm{~nm}$ with $200 \mathrm{kHz}$ linewidth is used as optical source. The light is modulated by a Mach-Zehnder modulator with output pulses of $10 \mathrm{~mW}$ peak power and 400 ns duration (either single or 127-bit Simplex-coded pulses), providing an attainable spatial resolution of $40 \mathrm{~m}$. In order to reduce the coherence Rayleigh noise $(\mathrm{CRN})$ produced by the narrowband optical source, we have applied wavelength averaging techniques, sweeping the wavelength of the laser over $0.5 \mathrm{~nm}$, and current dithering to the laser, which also helps to increase the SBS threshold.

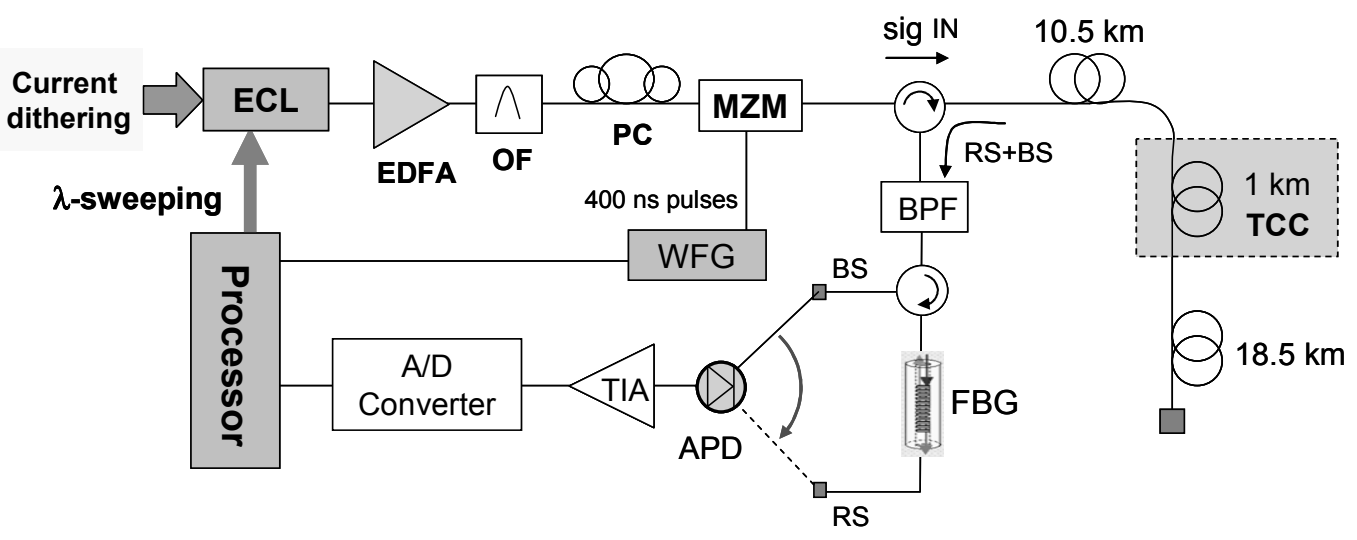

Figure 1. Experimental Setup (ECL: External cavity laser, OF: Optical Filter, PC: Polarization controller, MZM: Mach-Zehnder modulator, WFG: Waveform generator, TCC: Temperature-controlled chamber, BPF: Bandpass Filter, BS: Brillouin scattering, RS: Rayleigh scattering, FBG: Fiber Bragg grating, TIA: Transimpedance amplifier). 
The sensing fiber is composed by three spools of 10.5 $\mathrm{km}, 1 \mathrm{~km}$, and $18.5 \mathrm{~km}$ of dispersion-shifted fiber (DSF) for a total distance of $30 \mathrm{~km}$. The middle spool of fiber has been placed inside a temperature controlled chamber (TCC), which allows for a precise control of the fiber temperature. The TCC has been set to different temperature values ( $300 \mathrm{~K}$ and $343 \mathrm{~K}$ ) during the experiment.

At the receiver, the spontaneous anti-Stokes Brillouin component is separated from the Rayleigh signal through a narrowband fiber Bragg grating (FBG, $6 \mathrm{GHz}$ ) and an optical circulator, providing $28 \mathrm{~dB}$ band rejection. The optical signal is detected by using a $80 \mathrm{MHz}$ high-gain InGaAs avalanche photodiode (APD) followed by a high-gain transimpedance amplifier (TIA) and an analog-to-digital converter (ADC).

It is important to remark that wavelength averaging is needed only for Rayleigh measurements since spontaneous Brillouin scattering is not affected by coherent noise due to the random nature of the process. This feature allows us to use a narrowband FBG with only $6 \mathrm{GHz}$ reflected band allowing an effective spectral separation of the Rayleigh scattered signal and spontaneous anti-Stokes Brillouin component.

\section{RESULTS}

Figure 2 shows a comparison of the anti-Stokes Brillouin trace obtained by the conventional single-pulsed BDTS and the trace obtained by using 127-bit Simplex-coded BDTS, for a total number of 180,000 acquired traces in both cases. The noise reduction in the anti-Stokes Brillouin trace is clearly evident as a result of the application of Simplex coding. The SNR enhancement provided by 127-bit Simplex coding results to be greater than $7 \mathrm{~dB}$, being in good agreement with the theoretical prediction given by (3), estimating $G_{C O D}=7.54 \mathrm{~dB}$.

Note that, results have been obtained with the use of both current dithering and wavelength averaging techniques over

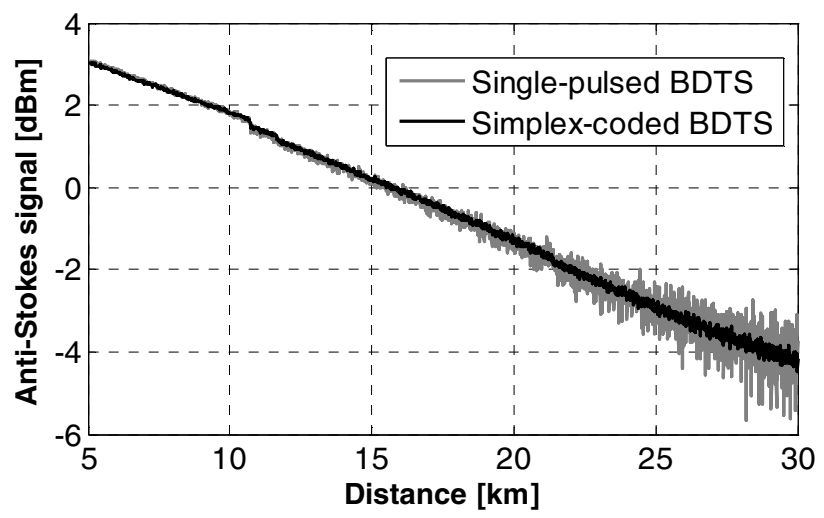

Figure 2. Spontaneous anti-Stokes Brillouin trace along $30 \mathrm{~km}$ sensing fiber. Curves for conventional and 127-bit Simplex-coded BDTS.

$0.5 \mathrm{~nm}$, reducing the coherent fading noise produced in Rayleigh measurements down to $0.4 \%$.

The distributed temperature profile along the sensing fiber is obtained through (2), where the room temperature $(300 \mathrm{~K})$ has been taken as a reference temperature. Figure 3 reports the temperature along the sensing fiber, when the TCC has been set at $343 \mathrm{~K}$, for both conventional and Simplex-coded BDTS. It can be clearly seen that the SNR enhancement provided by Simplex coding has a strong impact in the temperature accuracy of the sensor.

The achieved temperature resolution in both cases is shown in Figure 4. It has been estimated as an exponential fit of the standard deviation of the measured temperature distribution versus distance. For conventional-BDTS, a poor temperature resolution is achieved $(46.4 \mathrm{~K})$ at $30 \mathrm{~km}-$ distance because of the low peak pulse power $(10 \mathrm{~mW})$ used in the experiment; however, this is improved to $5.0 \mathrm{~K}$ due to the achieved SNR enhancement in temperature traces when using Simplex coding. At the fiber input, for both singlepulsed and Simplex-coded BDTS, the temperature resolution results to be better than $0.9 \mathrm{~K}$.

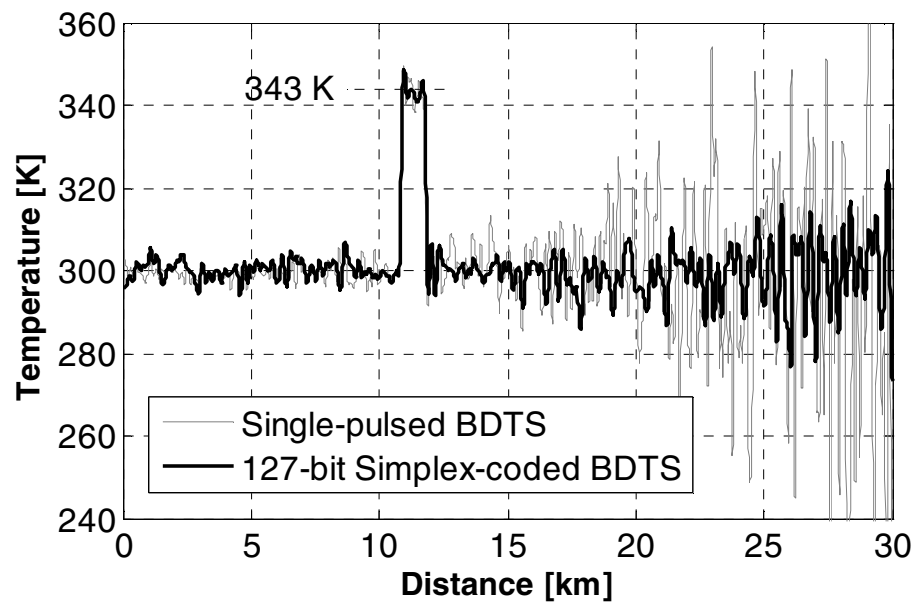

Figure 3. Temperature profile along $30 \mathrm{~km}$ sensing fiber. Curves for conventional and 127-bit Simplex-coded BDTS. The temperature of the fiber placed inside the TCC is $343 \mathrm{~K}$. 


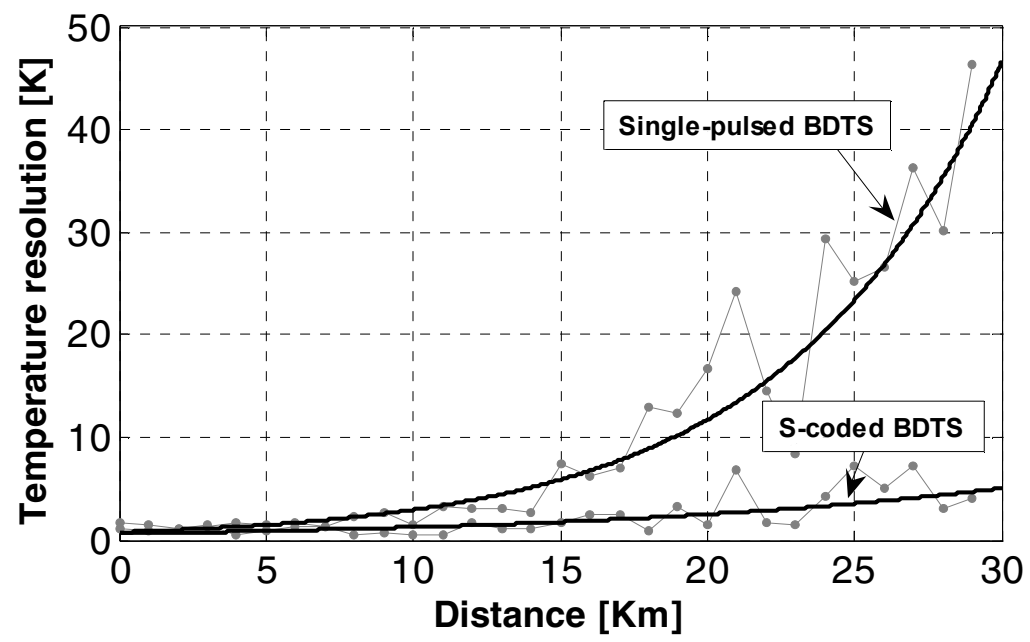

Figure 4. Temperature resolution for single-pulsed BDTS and 127-bit Simplex-coded BDTS.

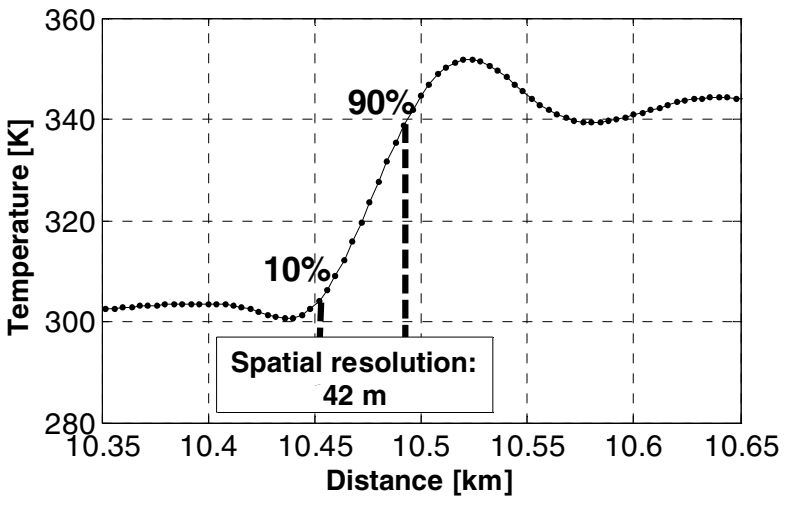

Figure 5. Achieved spatial resolution.

On the other hand, the actual spatial resolution achieved by the BDTS has been obtained by calculating the $10 \%$ to $90 \%$ response distance. Figure 5 reports the spatial resolution calculation, which results to be $42 \mathrm{~m}$ for both single and Simplex-coded BDTS.

It is important to remark that from the experiment we have detected that coding process results in a longer effective length for the BDTS with respect to the single-pulsed BDTS, producing a reduction of the stimulated Brillouin threshold value. This feature limits the maximum input peak power allowed when using coding techniques; nevertheless, coding techniques provide accurate temperature sensing at low peak power levels. We have found that when using 127-bit Simplex coded BDTS, it is possible to achieve a similar optimum temperature resolution than for single-pulsed BDTS, but using $\sim 15 \mathrm{~dB}$ lower input peak power.

\section{CONCLUSIONS}

In conclusion, we have proposed and experimentally demonstrated the feasibility of using coding technique in LPR-based BDTS for temperature resolution enhancement. Experimental results show that 127-bit Simplex coding offers $~ 7.1 \mathrm{~dB}$ SNR enhancement, allowing for $30 \mathrm{~km}$ sensing distance with a temperature and spatial resolution of $5.0 \mathrm{~K}$ and $42 \mathrm{~m}$, respectively, using only $10 \mathrm{~mW}$ pulse peak power at the fiber input. Results indicate that coding techniques can be successfully applied in BDTS as an alternative to optical pulse amplification, allowing for the use of low power optical sources and providing at the same time a high-performance cost-effective solution.

\section{REFERENCES}

[1] Nature Photonics, "Optical-fibre Sensors," Tech. Focus, vol. 2, pp. 143-158, 2008.

[2] S. M. Maughan, H. H. Kee and T. P. Newson, "Simultaneous distributed fibre temperature and strain sensor using microwave coherent detection of spontaneous Brillouin backscatter," Meas. Sci. Technol., vol 12, pp 834-842, 2001.

[3] P. C. Wait, K. De Souza, T. P. Newson., "A theoretical comparison of spontaneous Raman and Brillouin based fibre optic distributed temperature sensors," Opt. Commun., vol. 144, pp. 17-23, 1997.

[4] X. Bao, D. J. Webb, and D. A. Jackson, "Combined distributed temperature and strain sensor based on Brillouin loss in an optical fiber," Opt. Lett., vol. 19, no. 2, pp. 141-143, 1994.

[5] Y. T. Cho, M. Alahbabi, M. J. Gunning, T. P. Newson, "50-km single-ended spontaneous-Brillouin-based distributed-temperature sensor exploiting pulsed Raman amplification," Opt. Lett., vol. 28, pp. 1651-1653, 2003.

[6] K. De. Souza and T. P. Newson, "Improvement of signal-to-noise capabilities of a distributed temperature sensor using optical preamplification," Meas. Sci. Technol., vol.12, pp. 952- 957, 2001.

[7] M. D. Jones, "Using Simplex codes to improve OTDR Sensitivity," IEEE Photon. Technol. Lett., vol. 15, pp. 822-824, Jul. 1993.

[8] D. Lee et al, "Analysis and Experimental Demonstration of Simplex Coding Technique for SNR Enhancement of OTDR," In Proc. IEEE LTIMC, pp. 118-122, New York, USA, Oct. 2004.

[9] P. C. Wait, T. P. Newson., "Landau Placzek ratio applied to distributed fiber sensing," Opt. Commun., vol. 122, pp. 141-146, 1996.

[10] J. Park et al, "Raman-based distributed temperature sensor with Simplex coding and link optimization," IEEE Photon. Technol. Lett., vol. 18, pp. 1879-1881, 2006. 